\title{
DER THROUGHPUT-OUTPUT-NEXUS IN DER EMPIRISCHEN VERWALTUNGSWISSENSCHAFT
}

\author{
Von Michael W. Bauer, Konstanz*
}

\section{Einführung}

Die empirische Verwaltungswissenschaft ist eine etablierte Teildisziplin der Politikwissenschaft ${ }^{1}$. Als Verwaltungsreformwissenschaft untersucht sie den Wandel von öffentlichen Organisationen und fragt insbesondere nach den Auswirkungen bestimmter Reformstrategien in bestimmten institutionellen Kontexten und Akteurskonstellationen. Die Hauptaufgabe der Verwaltungswissenschaft als Verwaltungsreformwissenschaft wird gemeinhin darin gesehen, über eine Erfassung und A nalyse von staatlichem Organisationswandel, Rückschlüsse darauf zu ziehen, wie sich spezifische Reformen im öffentlichen Sektor auf die (effiziente) Erzeugung und letztlich auf die (intendierte) Ziel errei chung staatlicher Dienstleistungen auswirken. ${ }^{2}$

Akzeptiert man als essentielle Aufgabe der Verwaltungswissenschaft die verlässliche Erfassung des Zusammenhangs zwischen Organisationsreform und Ergebnis, tritt ein erstaunliches Defizit zutage: Trotz jahrzehntelanger Bemühungen der empirischen Verwaltungsforschung verfügen wir nur in sehr unzureichendem U mfang über systematisches Wissen über die Zusammenhänge zwischen dem Wandel von öffentlichen Organisationen und den tatsächlichen Resultaten, die di ese organisationalen Veränderungen ursächlich bewirken. In anderen Worten, obwohl Verwaltungsreformen seit vielen J ahren zentrale Untersuchungsobjekte sind, bleibt unser Wissen deskriptiv und überwiegend prozessorientiert, unsere Hypothesen über Zusammen-

* Der Autor dankt Prof. Dr. Matthias Ruffert und Dipl. Verw. Wiss. J ulia Fleischer für hilfreiche Kommentare.

1 F. Scharpf, Verwaltungswissenschaft als Teil der Politikwissenschaft, in: ders. (Hrsg.), Planung als politischer Prozess. Aufsätze zur Theorie der planenden Demokratie, 1973, S. 9; J. Hesse (Hrsg.), Politikwissenschaft und Verwaltungswissenschaft, 1982. W. Jann, Politikwissenschaftliche Verwaltungsforschung, in: K. von Beyme (Hrsg.), Politikwissenschaft in der Bundesrepublik Deutschland, 1996; A. Benz, Status und Perspektiven der politikwissenschaftlichen Verwaltungsforschung, Die Verwaltung 36 (2003), S. 361. Vgl. aktuell J. Bogumil / W. J ann / F. Nullmeier, Perspektiven der politikwissenschaftlichen Verwaltungsforschung, in: dies. (Hrsg.), Politik und Verwaltung, PVS-Sonderheft 37 (2006), S. 9.

2 J. B ogumil / W. J ann, Verwaltung und Verwaltungswissenschaft in Deutschland. Einführung in die Verwaltungswissenschaft, 2005. 
hänge weitgehend ad hoc, die Akkumulation von Erkenntnissen über Fallgrenzen hinweg unzureichend und unser theoretisches Verständnis von Verwaltungsreformen und ihren Effekten mithin begrenzt ${ }^{3}$. Als Konsequenz daraus fällt es empirischen Verwaltungswissenschaftlern gewöhnlich schwer, das Verdikt der mangelnden theoretischen Reife ihrer Teildisziplin zu entkräften.

Dieser Aufsatz untersucht, ob die Verwaltungswissenschaft gegebenenfalls über die Rezeption policyanalytischer Konzepte ihre Prognosefähigkeit verbessern und damit ihr zentrales Theoriedefizit im Hinblick auf den Reform-Ergebnis-Zusammenhang abbauen kann. Zur Beantwortung dieser Frage wird im folgenden Abschnitt kurz auf das Verhältnis von Verwaltungswissenschaft und Policy-Analyse eingegangen und sodann eine si mple Strategie entwickelt, wie policyanalytische Fragestel lungen für die Analyse von Effekten von Verwaltungsreformen fruchtbar gemacht werden könnten. Am Beispiel der jüngsten Verwaltungsreformen in der Europäischen Kommission wird anschließend illustriert, wie die entwickel te Strategie Anwendung finden könnte. Schließlich werden analytischer Mehrwert sowie Implikationen des vorgeschlagenen Ansatzes für die weitere Verwaltungsforschung in diesem Kontext diskutiert.

\section{Verwaltungswissenschaft und Policy-Analyse}

Die moderne Policy-A nalyse sucht nach den Eigengesetzlichkeiten spezifischer Politiken mit dem Gerüst traditioneller politikwissenschaftlicher Fragestel lungen und fragt insbesondere nach dem Zusammenhang zwischen politischen Institutionen, politischem Prozess und Politikinhalt ${ }^{4}$. Der Politikbegriff, welcher der Policy-Analyse dabei zugrunde liegt, kann als Prozess beschrieben werden, ,in dem lösungsbedürftige Probleme artikuliert, politische Ziel e formuliert, alternative Handlungsmöglichkeiten entwickelt und schließlich als verbindliche Festlegung gewählt werden“5. Angesichts dieser Zielsetzung ${ }^{6}$ darf man die Policy-Analyse im Vergleich mit der Ver-

3 Das gilt nicht nur für Untersuchungen, die auf Deutschland bezogen sind, sondern ist ein internationals Defizit. Vgl. C. Pollitt/G. Bouckaert, Public Management Reform. A Comparative Analysism, 2. Aufl. 2004; J. Olsen / G. Peters (Hrsg.), Lessons from Experience. Experimental Learning in Administrative Reforms in Eight Democracies, 1996; G. Peters/D. Savoie (Hrsg.), Taking Stock. Assessing Public Sector Reforms, 1998.

4 A. Windhoff-Héritier, Policy-Analyse. Eine Einführung, 1987; M. Schmidt, Policy-Analyse, in: A. Mohr (Hrsg.), Grundzüge der Politikwissenschaft, 1995, S. 566; A. Héritier, Policy-Analyse. Elemente der K ritik und Perspektiven der Neuorientierung, in: ders. (Hrsg.), Policy-Analyse. K ritik und Neuorientierung, PVS-Sonderheft, 1993, S. 9; M. Howlett/ M. Ramesh, Studying Public Policy. Policy Cycles and Policy Subsystems, 2003.

5 Scharpf (FN 1), S. 27. 
waltungswissenschaft vom Anspruch her als das umfassendere Projekt bezeichnen. Gleichwohl ist offensichtlich, dass es zwischen diesen beiden Teildisziplinen Überlappungen in den Erkenntnisinteressen und Untersuchungsobjekten gibt, denn die Policy-Analyse als die Untersuchung des "arbeitenden Staates“ kommt ohne die Einbeziehung der staatlichen Verwaltung fast nie aus ${ }^{7}$. Es ist auch festzustellen, dass Verwaltungswissenschaftler in jüngster Zeit ihre Analysen immer mehr auf einzelne Politikfelder fokussieren ${ }^{8}$. Häufig arbeiten einzelne Wissenschaftler denn auch politikfeldspezifisch als Verwaltungsreformwissenschaftler und als Policy-Forscher. Dennoch können die Kernbereiche der beiden Subdisziplinen de facto relativ unproblematisch unterschieden werden. Das nachfolgende Schaubild ist dabei hilfreich. Die Analyseel emente von Verwaltungswissenschaft und Policy-Analyse lassen sich nämlich in der Sprache der Systemtheorie, welche beide Teildisziplinen insbesondere in ihren Entstehungsphasen erheblich geprägt hat, vereinfacht darstellen ${ }^{9}$. Verwaltungsreformwissenschaftler konzentrieren sich in der Praxis, so die Implikation des Schaubilds, überwiegend auf die Analyse von Inputs und Throughputs (also eher auf den Inhalt der Ellipse A) ${ }^{10}$. Im Gegensatz dazu sind Policy-Forscher mehr an Fragen des Outputs und mittelbaren Outcomes interessiert (al so eher den Inhalt der Ellipse B). Verwal tungswissenschaftler konzentrieren sich al so mehr auf den Reformprozess, die Substanz, das Timing und die Konsequenzen der Reform im staatlichen Apparat; sie fragen mithin nach den Auswirkungen von Reformen auf dieThroughputs. Policy-Forscher hingegen untersuchen Policy-Wandel und fragen nach Einwirkungen von Änderungen der Inputs und Throughputs auf den Policy-Output und (sofern möglich) auf den Policy-Outcome. Noch pointierter formuliert könnte man sagen, die Verwaltungswissenschaftler sehen in den Verwaltungsreformen selbst ihre abhängige Variable, also das, was erklärt werden soll. Wohin-

6 Ursprünglich war Policy-Analyse, Policy Sciences, konzipiert als ein Projekt einer neuen problemorientierten „praktischen Philosophie“, welches sich den grundlegenden Problemen des modernen Menschen in der Gesel Ischaft widmen wollte und diese Probleme theoretisch wie interdisziplinär angehen sollte. D. Lerner / H. L asswell (Hrsg.), The Policy Sciences. Recent Developments in Scope and Method, 1951; H. Lasswell, A pre-view of policy sciences, 1971.

7 K. König/J. Beck (Hrsg.), A rbeitender Staat: Studien Zur Regierung und Verwaltung. Klaus König zum sechzigsten Geburtstag, 1995.

8 Das wurde beispielsweise unlängst auf der Tagung "Verwaltung in Politikfeldern“ der Sektion "Staatslehre und politische Verwaltung“ der Deutschen Vereinigung, 31. 5. - 1. 6. 2007 an der Ruhr-Universität Bochum augenfällig.

9 D. Easton, A Systems Analysis of Political Life, 1965; N. Luhmann, Zweckbegriff und Systemrationalität. Über die Funktion von Zwecken in sozialen Systemen, 1968.

10 Input, Output und Outcome oder I mpact sind mittlerweile gängige Begriffe. Der Terminus Throughput hingegen wird weniger häufig benutzt. Er bezeichnet die internen Organisationsmerkmale, die den Input tatsächlich in einen organisational en Output verwandeln. Vgl. FN 9. 
gegen die Policy-Forscher im gleichen Kontext an Reformen eher als unabhängige Variablen interessiert sind. Sie konzeptionalisieren Reformen als Erklärungsfaktoren für den sich verändernden Policy-Outcome, also das Politikergebnis ${ }^{11}$. Hinter dieser beobachtbaren "Arbeitsteilung“ steht sel bstredend kein Naturgesetz, sondern eher eine Tendenz, die wohl viel mit pragmatischer Sel bstbeschränkung auf individueller Ebene der Forscher zu tun hat.

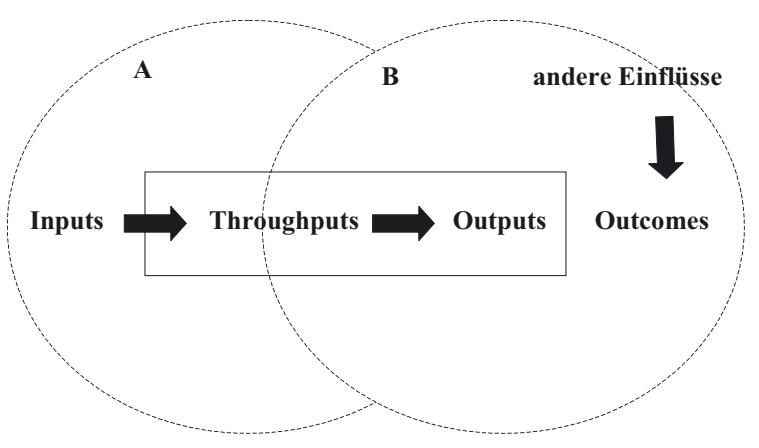

\section{Von der Policy-A nalyse lernen?}

Die Diagnose lautet also, dass die politikwissenschaftliche Verwaltungswissenschaft faktisch ihr primäres Forschungsfeld in der deskriptiven Rekonstruktion von Reformvorgängen sieht und erhebliche D efizite im Hinblick auf die Generierung von allgemeingültigen Aussagen über den $\mathrm{Zu}$ sammenhang von spezifischen Reformen und deren Folgen aufweist. Trifft diese Diagnose zu, stellt sich die Frage, wie nun eine Optimierung im Sinne der Erzeugung von systematischen und theoriegeleiteten Aussagen über den zentralen Zusammenhang zwischen Throughput-Veränderungen auf der einen Seite und Output-Resultaten von Verwaltungsreformen auf der anderen ermöglicht werden kann.

Wünschenswert wäre zweifelsohne die Verfolgung vergleichender Forschungsdesigns, die über lange Zeiträume hinweg (und zwar vor und nachdem Reformen stattfinden), Outputs und Outcomes erfassen, Fremdeinflüsse isolieren und damit die Effekte von Reformen zurechenbar machen ${ }^{12}$.

11 Zu den Begrifflichkeiten vgl. R. Schnell / P. Hill / E. Esser, Methoden der empirischen Sozialforschung, 2005, insbesondere K apitel 5; H. Esser, Soziologie. Allgemeine Grundlagen, 1993.

$12 \mathrm{Vgl}$. D. Jahn, Einführung in die vergleichende Politikwissenschaft, 2006; S. K ropp / M. Minkenberg (Hrsg.), Vergl eichen in der Politikwissenschaft, 2005. 
Leider ist es nicht nur analytisch äußerst schwierig, mit derart komplexen Kausalketten über lange Zeiträume zurechtzukommen ${ }^{13}$. Gewöhnlich stehen die hiefür notwendigen Ressourcen dem einzelnen Forscher auch gar nicht zur Verfügung; oder aber es sind zeitnahe Bewertungen gefragt, die langfristige Datenerhebungs- und Auswertungsstrategien von vornherein ausschließen. Eine realistische und pragmatische Möglichkeit, die dem Forscher allerdings sehr wohl zur Verfügung steht, besteht in der Theoretisierung des zentralen Zusammenhangs zwischen Organisationsreform und Reformergebnis, oder dem, was man als Throughput-Output-Nexus bezeichnen könnte, unter Verwendung policyanalytischer Kategorien und K onzepten ${ }^{14}$. Notwendig hierfür ist al lerdings ei ne Vereinfachung und Konzentration der Forschungsfrage auf eben diesen Kernzusammenhang ${ }^{15}$. Die wesentliche Frage muss dann ex-ante lauten, wie wirkt sich eine Veränderung der Interna einer Organisation auf den Policy-Output und damit auf den mittelbaren Produktionsprozess einer bestimmten Politik aus? Mit anderen Worten, man muss eine Theoretisierung der durch eine Verwaltungsreform betroffenen Besonderheiten einer Organisation vornehmen und die Implikationen dieser Änderungen auf den relevanten Policy-Output radikal in den Mittelpunkt der Analyse stellen. So eröffnet sich eine real istische und pragmatische Option, analytischen Zugriff auf Output-Resultate zu gewinnen und damit gegebenenfalls die Grundlage dafür zu schaffen, theoriegeleitete und empiriegesättigte Aussagen über die zu erwartenden Effekte von Organisationswandel zu generieren.

Bildlich gesprochen sollte man den "organisatorischen Nerv" einer Verwaltung, das heißt jenen Organisationsteil, der den Throughput-OutputNexus entscheidend prägt, mithilfe policyanalytischer Konzepte zu identifizieren suchen, um dann im nächsten Schritt die durch eine bestimmte Reform bewirkten Änderungen auf diesen zentralen - und nur theoretisch, das heißt im Lichte einer besonderen Fragestell ung bestimmbaren - organisatorischen Nerv zu erfassen und auf der Grundlage empirischer Beobachtungen zu bewerten. Wie das im Einzelfall aussehen könnte, lässt sich am besten an einem konkreten Beispiel illustrieren. Das soll im folgenden Abschnitt am Beispiel der jüngsten Verwaltungsreform in der Europäischen Kommission geschehen. Zuvor gilt es allerdings noch einigen möglichen Missverständnissen vorzubeugen.

13 Gemeint sind hier reformunabhängige Veränderungen, die auch den Output einer Organisation beeinflussen wie interne Prioritätensetzung, Nachfrage von politischen Prinzipalen, Kontextfaktoren usw.

14 Synergieeffekte zwischen den Teildisziplinen sind aus dieser Perspektive sehr wohl möglich. Vgl. F. J anning, K oexistenz ohne Synergieeffekte? Über das Verhältnis zwischen Policy-Forschung und Verwaltungswissenschaft, in: Bogumil / J ann / Nullmeier (FN 1), Politik und Verwaltung. PVS-Sonderheft 37, 2006, S. 77.

15 G. King/R. Keohane/ S. Verba, Designing Social Inquiry, 1994; J. Gerring, Case Study Research: Principles and Practices, 2007. 
Da sich Verwaltungsforscher ihrem Gegenstand vermehrt über die Perspektive bestimmter Politikfelder nähern, und damit die klassische Herangehensweise der Policy-Analyse im Ansatz bereits nachahmen, werden mit dem Vorschlag, verwal tungswissenschaftl iche Reformanalysen systematisch auf den politikrelevanten Throughput-Output-Nexus zu fokussieren, nur fol gerichtig die Konsequenzen dieser bereits stattfindenden Übernahme des policyanalytischen Forschungsansatzes für die Verwaltungsreformwissenschaft gezogen ${ }^{16}$.

Es wird ferner häufig als Stärke der gegenwärtigen Verwaltungswissenschaft gesehen, dass diese über ihre Phänomene eine beschreibende Ti efe und damit ein breites Verständnis zu entwickeln vermag ${ }^{17}$. Legt man als Standard allerdings nicht retrospektive Rekonstruktion, sondern Produktion von fallübergreifenden, möglichst allgemeingültigen Aussagen an, ist es einer Sozialwissenschaft nicht möglich, bei einer analytischen Fallbeschreibung stehen zu bleiben ${ }^{18}$. Die Produktion von einzelfallunabhängiger Erkenntnis muss das anzustrebende Ziel bleiben, sofern man sich nicht dauerhaft mit einer propädeutischen Verwaltungslehre bescheiden will. Das heißt aber auch, dass man sich der Herausforderung der Modellierung, also einer theoriegeleiteten Vereinfachung der Zusammenhänge, die die Grundlage für die oben vorgeschlagene Strategie zur Erhellung des Throughput-Output-Nexus bildet, nicht einfach mit dem Verweis auf den damit einhergehenden Verlust an beschreibender Tiefenschärfe entziehen kann. Ein wesentlicher Aspekt von theoretischer Erkenntnis besteht ja gerade in der damit erreichbaren Reduktion von infinit komplexer Realität.

Ausdrücklich muss schließlich betont werden, dass das vorgeschlagene Vorgehen per se in keiner Weise die methodischen Probleme der Verwaltungswissenschaft als Sozialwissenschaft lösen kann. Wie alle Sozialwissenschaften hat auch die Verwaltungswissenschaft ihren Untersuchungsgegenstand in den unvollkommenen und selbstreferenziellen Artefakten sozialer Organisation und kann daher in der Regel keine Versuchsanordnungen unter Laborbedingungen zur Beantwortung ihrer Fragestellung erzeugen und nutzen ${ }^{19}$. Es geht also bei dem Vorschlag, den Throughput-

16 Auf das Potenzial der Policy-A nalyse zur Konsolidierung einer „positiven Theorie der Verwaltung“ weisen auch J. B ogumil, W. J ann und F. Null meier hin; sie bleiben jedoch grundsätzlich eher kritisch über den zu erreichenden Mehrwert und geben wenig Hinweise, wie genau die gängigen policy-analytischen Konzepte (Policy-Phasen-Zyklus, Instrumentenklassifikationen, Advocacy-Koalition etc.) zur Verwaltungsanalyse herangezogen werden können. Vgl. Bogumil/Jann/ Nullmeier (FN 1), S. 17.

17 G. Schuppert, Verwaltungswissenschaft. Verwaltung, Verwaltungsrecht, Verwaltungslehre, 2000.

18 H. Brady/D. Collier (Hrsg.), Rethinking Social Inquiry. Diverse Tools, Shared Standards, 2004; G. Goertz, Social Science Concepts. A U ser's Guide, 2006. 
Output-Nexus in den Mittelpunkt zu stellen und damit verstärkt auf policyanalytische Konzeptionalisierungen bei der Identifikation von Verwaltungsreformeffekten zurückzugreifen, nicht um die (illusorische) Ü berwindung der Defizite der Verwaltungswissenschaft als Sozialwissenschaft. Sondern viel bescheidener lediglich darum, auf ungenutztes und praktikables Potenzial zu verweisen, wie angesichts der bekannten strukturellen Probleme sowie den notorisch knappen Forschungsressourcen kurzfristig theoretisch höherwertige Reformanalysen erarbeitet werden können.

\section{D ie Verwaltungsreform der E uropäischen K ommission}

In diesem Abschnitt soll ein Beispiel gegeben werden, wie durch die Konzentration auf den Throughput-Output-Nexus mithilfe policyanalytischer Konzepte, verwaltungswissenschaftliche Reformanalysen im Hinblick auf die Generierung von Aussagen über Reformeffekte optimiert werden können.

Die Europäische Kommission ist ein zentraler Akteur im Politikprozess der EU; Änderungen ihrer administrativen Kapazitäten und ihrer Rolle sind daher bedeutsam ${ }^{20}$. Über die jüngste Verwaltungsreform - die sog. Kinnock Reform - gibt es zwar bereits eine anschauliche verwaltungswissenschaftliche Literatur ${ }^{21}$. Aber die Auswirkungen, die diese Reform auf den Politikprozess der EU hat, sind noch weithin unerforscht. Mithin weist die verwaltungswissenschaftliche Analyse der jüngsten Kommissionsreform exakt das oben angesprochene generelle Defizit verwaltungswissenschaftlicher Reformanalysen auf, wonach die Organisationsveränderungen selbst und nicht deren Auswirkungen im Mittelpunkt der Forschungsanstrengungen stehen. Die eigentliche Herausforderung ist also auch im Fall der Europäischen Kommission, den Throughput-Output-Nexus zu fokussieren und theoriegeleitet die Frage nach den Auswirkungen der jüngsten Reform zu stellen.

19 J. Gerring, Social Science Methodology. A Criterial Framework, 2001; A. George/ A. B ennett, Case Studies and Theory Development in the Social Sciences, 2005.

20 N. Nugent, The European Commission, 2001.

21 H. Kassim, A Historic Accomplishment. The Prodi Commission and Administrative Reform, in: D. G. Dimitrakopoul os (H rsg.), The Changing European Commission, 2004, S. 33; ders., The K innock Reforms in Perspective: Why reforming the Commission is an Heroic, But Thankless Task, in: Public Policy and Administration 19 (2004), S. 25; R. Levy, European Commission Overload and the Pathology of Management Reform: Garbage Can, Rationality and Risk Aversion, in: Public Administration 84 (2006), S. 423; L. Metcalfe, Reforming the Commission: Will Organisational Efficiency Produce Effective Governance?, in: J ournal of Common Market Studies 38 (2000), S. 817; D. Spence/A. Stevens, Staff and Personnel Policy in the Commission, in: D. Spence/ G. Edwards (H rsg.), The European Commission, 2006, S. 173. 
Um nun die Frage zu beantworten, welche Konsequenzen der jüngste Organisationswandel für die Rolle der Kommission im Kontext des europäischen Politikprozesses birgt, ist zunächst einmal zu fragen, worin denn diese Rolle der Kommission als Verwaltung theoretisch überhaupt besteht. Es ist wahrscheinlich, dass man für eine beliebige Verwal tung mehrere solcher Rollen (je nach Fokus oder Stand der Debatte) konzeptionalisieren kann. I dealerweise würde man dann die jeweils erwartbaren Implikationen (im Hinblick auf den aktuellen Organisationswandel) für alle einschlägigen Organisationsrollen oder -funktionen trennscharf herausarbeiten und im Vergleich zueinander im Fokus des Throughput-Output-Nexus erfassen und bewerten.

Für die Europäische Kommission können drei solcher Funktionen ausgemacht werden: Politikinnovation, Wettbewerbssicherung und Implementationsüberwachung ${ }^{22}$. Da es in diesem A bschnitt allein um die beispiel hafte Illustration eines theoriegeleiteten Analyseansatzes geht, darf man sich im Folgenden auf die Rolle der Kommission bei der innovativen Entwicklung europäischer Politiken konzentrieren.

Die zentrale These ist hier, dass die Kommission - nicht zul etzt durch ihr Initiativmonopol - das Agenda-Setting im EU Politikprozess dominiert ${ }^{23}$. Präzisiert man diese Dominanz, wird deutlich, dass das wesentliche Element in diesem Kontext die Fähigkeit der Kommission ist, als "PolitikEntrepreneur" oder „politischer Unternehmer" aufzutreten ${ }^{24}$. Das hei ßt, durch clevere, manchmal opportunistische Schachzüge versteht es die Kommission, Kompromisse vorzubereiten, aber auch über lange - oder zumindest über längere Zeit - an bestimmten Politiklösungen - auch gegenüber Widerstand der Mitgliedstaaten der Union - festzuhal ten und deren Umsetzung hartnäckig zu verfol gen ${ }^{25}$.

Oft wird mit dem policyanalytischen Konzept des politischen Unternehmers im Hinblick auf die Rolle der Kommission sehr vage umgegangen.

$22 \mathrm{~L}$. Hooghe, The European Commission and the Integration of Europe. Images of Governance, 2001; M. W. Bauer, Diffuse Anxieties, Deprived Entrepreneurs. Commission Reform and Middle Management, in: ders. (Hrsg.), Reforming the European Commission, Special Issue of the J ournal of European Public Policy 15 (2008), (im Erscheinen).

$23 \mathrm{G}$. Peters, Agenda-Setting in the European Community, Journal of European Public Policy 1 (1994), S. 9.

24 L. Cram, Calling the Tune without Paying the Piper? Social Policy Regulation. The Role of the Commission in European Community Social Policy, Policy and Politics 21 (1993), S. 135; A. Héritier, Policy-Making by Subterfuge: Interest Accommodation, Innovation and Substitute Democratic Legitimation in Europe - Perspectives from distinct Policy Areas, J ournal of European Public Policy 4 (1997), S. 171; M. W. Bauer, Limitations to Agency Control in EU Policy-Making - the Commission and the Poverty Programmes, J ournal of Common Market Studies 40 (2002), S. 381.

25 M. W. Bauer / M. Hartlapp, Explaining Patterns of Expansive Usage of Delegated Power. The European Commission and Annulment Proceedings, Journal of Common Market Studies (2007), (in Begutachtung). 
Schaut man sich die ei nschlägigen Arbeiten aber genauer an, wird klar, dass das „politische Unternehmertum“ in der Kommission eine „individuelle Aufgabe“ ist und die eigentlichen „Unternehmer" zwischen dem TopManagement und den gewöhnlichen Mitarbeitern sitzen. Gemeint sind die Abteilungsleiter innerhalb der Kommission ${ }^{26}$, die einen im Vergleich mit ähnlichen Funktionen in nationalen Verwaltungen ungleich größeren Einfluss ausüben. Die Abteilungsleiter sind Individuen mit langjähriger Arbeitserfahrung, Spezialisten auf ihrem Fachgebi et, die ein Team von Mitarbeitern anleiten. Sie sitzen an der entscheidenden Stelle, wo kommissionsintern inhaltliche Expertise auf politisches Management trifft. Unterhalb der Abteilungsleiter fehlen der politische Überblick und die Positionsmacht und oberhalb der Abteilungsleiter sind die Positionen bereits zu stark politisch bzw. mit Prozessmanagement beschäftigt, um tatsächlich die technischen Einzelheiten eines Dossiers zu überschauen und produktiv zur unerlässlichen Kompromissbildung nutzen zu können. Auf die Abteilungsleiter kommt es an, einen Vorschlag so einzufädeln, dass er im EU-Politikprozess überhaupt Überlebenschancen hat ${ }^{27}$. Es ist natürlich eine Vereinfachung, aber wenn man so etwas wie eine Mikrofundierung von politischem Unternehmertum in der Kommission anstrebt, also Anreizstrukturen auf der individuellen Akteursebene als Voraussetzung für die Aufstellung einer vollständigen theoretischen Erklärung ansieht, kommen eigentlich nur die Abteilungsl eiter als die rel evante A kteursgruppe in Frage ${ }^{28}$.

Damit ist verwaltungswissenschaftlich der zentrale „Organisationsnerv“ freigel egt, der policy-theoretisch interessiert. Denn, wenn die Reformeffekte in der Kommission analysiert werden sollen, und policyanalytisch die wichtigste Rolle der Kommission als Politikunternehmer bestimmt ist, dann kann man nach den besonderen Auswirkungen der Veränderungen im K ontext der K innock Reform auf die Abteilungsl eiter fragen und entsprechende Rückschlüsse ziehen. Die zentrale Forschungsfrage lautet damit: Was sind die Auswirkungen der jüngsten Verwaltungsreformen auf die Abteilungsl eiter in der Kommission? Um diese Frage beantworten zu können, müssen im nächsten Schritt kurz die wesentlichen Elemente der Kinnock Reform erläutert werden.

26 In der Diktion der Europäischen Kommission die „Heads of Unit“ vgl . die Ausführungen der Kommission auf ihrer homepage unter: http://ec.europa.eu/reform/ 2002/index_en.htm (12. 8. 2007).

27 M. Cini, The European Commission. Leadership, organisation and culture in the EU administration, 1996, S. 193; Nugent (FN 20), S. 242.

$28 \mathrm{Vgl}$. zur Problematik der Mirkofundierung und zur Akteursperspektive in theoretischen Erklärungen $\mathrm{D}$. B raun, Theorien rationalen $\mathrm{H}$ andel ns in der Politikwissenschaft. Einekritische Einführung, 1999; Esser (FN 11). 


\section{Die E uropäische K ommission als Reformnachzügler}

Die Geschichte der Verwaltungsreformen in der Kommission ist eine $\mathrm{Ge}$ schichte der verpassten Gel egenheiten ${ }^{29}$. Die Probleme an sich sind bereits seit Ende der 1970er Jahre bekannt. Aber erst der Luxemburger J acques Santer hat sie als Präsident der Kommission Ende der 1990er ins Zentrum einer Reformoffensive gestellt - und ist grandios gescheitert. ${ }^{30}$ Allerdings hat der erstmalige Rücktritt einer gesamten Europäischen Kommission der zwar nicht ursächlich mit dem Scheitern der Verwaltungsreformbemühungen in Verbindung gebracht werden kann, der aber sehr wohl Organisationsprobleme betraf, welche durch diese Reformen gelöst werden sollten das vorherrschende Problembewusstsein immens gesteigert. Santers Nachfolger Romano Prodi und mit inm sein Reformkommissar Neil Kinnock waren sozusagen unter produktivem Erfolgszwang. Das Ergebnis, die so genannte Kinnock Reform, wurde zwischen 2000 und 2004 entwickelt und umgesetzt. Es gab vier Reformkapitel Personalmanagement, Strategische Planung und Steuerung, Finanzmanagement sowie berufsethische Fragen. ${ }^{31}$ Man kann die Reform folgendermaßen zusammenfassen: Von einer eher kontinental geprägten Verwaltungskultur wurde die Kommission an einem Verwaltungsmodell à la New Public Management ausgerichtet. Das war sicherlich überfällig, denn die K ommission als Verwaltung hat ihre Managementstrukturen - trotz erheblichem Aufgabenzuwachs und Aufgabenwandel - in den ersten $40 \mathrm{~J}$ ahren ihres Bestehens faktisch nicht verändert. Leider kann an dieser Stelle auf die einzel nen Reformmaßnahmen nicht im Detail eingegangen werden ${ }^{32}$. Gesagt werden kann aber, dass die Reform unter Experten durchaus als ",historische“ Veränderung angesehen wird. Es gibt jedoch wenig I nformation darüber, wie man intern mit den Neuerungen tatsächlich zurechtkommt ${ }^{33}$. Deutlich ist allerdings, dass insbesondere die neue Personal politik und der gesamte Komplex der strategischen Planung und Steuerung tiefgreifende Auswirkungen auf die Arbeit der Abteilungsleiter haben. So beinhaltet die neue Personalpolitik neue Beförderungsregelungen, neue strukturierte individuelle Zielvereinbarungen und vieles

29 M. W. Bauer, The Politics of Reforming the European Commission Administration, in: M. W. Bauer / C. K nill (Hrsg.), Management Reforms in International Organizations, 2007, S. 54.

$30 \mathrm{~J}$. Peterson, The Santer Era: The European Commission in N ormative, Historical and Theoretical Perspective, J ournal of European Public Policy 6 (1999), S. 65.

$31 \mathrm{~N}$. Bearfield, Reforming the European Commission. Driving Reform from the Grassroots, Public Policy and Administration 19 (2004), S. 13. Vgl. auch das Weisbuch zur Reform sowie die folgenden Umsetzungsberichte auf der Homepage der Europäischen Kommission unter http://ec.europa.eu/reform/index_de.htm (12. 8. 2007).

$32 \mathrm{Vgl}$. auch das Weisbuch zur Reform sowie die folgenden Umsetzungsberichte auf der Homepage der Europäischen Kommission unter http://ec.europa.eu/reform/ index_de.htm (12. 8. 2007).

33 Bauer (FN 22). 
mehr. Alles Elemente, die die Rolle der Abteilungsleiter als Personalmanager verstärken. Ähnlich sieht es im Bereich der strategischen Planung und Steuerung aus. Auch hier muss der Abteilungsl eiter die Hauptlast der jüngsten Änderungen tragen. A bteilungsziele müssen ex-ante festgel egt werden, diese Ziele sind auf die einzel nen Gruppen bzw. Mitarbeiter herunterzubrechen, entsprechende Erfolgs- und Prozessindikatoren müssen konstruiert werden, vierteljährige Prozessverlaufs- und Ergebnisberichte sind zu erstellen, ex-post Evaluierungen durchzuführen und vieles mehr ${ }^{34}$. Kurzum, keine andere Position wurde von der Reform so stark verändert wie die des Abteilungsleiters. Die zentrale empirische Frage zur Erhellung des Throughput-Output-N exus ist also, wie wirken sich diese besonderen Organisationsveränderungen auf die individuelle Kapazität der Abteilungsleiter aus, ihre Rolle als politische Unternehmer auszufüllen? Prinzipiell stehen zur Beantwortung dieser Frage alle herkömml ichen qualitativen oder quantitativen Methoden zur Verfügung. Im Folgenden sollen kurz die wesentlichen E inblicke aus einer standardisierten U mfrage unter Abteilungsl eitern in der Kommission vorgestellt werden.

\section{A bteilungsleiter und deren Wahrnehmung der Reformauswirkungen: E mpirische Beobachtungen}

Der Survey, auf den hier Bezug genommen wird, wurde 2006 durchgeführt. Befragt wurden nur Abteilungsleiter in der Kommission mit Politikentwicklungsaufgaben. Sie wurden über ihre persönliche Wahrnehmung der Auswirkungen der Reform im individuellen Arbeitsumfeld befragt ${ }^{35}$. Die Details interessieren hier nicht weiter. Es konnte jedoch festgestellt werden, dass die große Mehrheit der Abteilungsleiter nach wie vor ein Selbstverständnis ihrer Rolle als „politische Unternehmer" hat und dass die Kinnock Reform ihre Kapazität, als solche politischen Unternehmer und Politikinnovatoren zu handeln, schmerzlich schmäl ert ${ }^{36}$.

\footnotetext{
34 Siehe die Beiträge in Bauer (Hrsg), FN22.

35 In der Kommission arbeiten rund 25.000 Beamte bzw. Angestellte. Sie hat 40 Generaldirektionen oder Dienste. Aber nur 13 davon sind so Generaldirektion mit Politikentwicklungsaufgaben wie DG Strukturpolitik, DG L andwirtschaft, DG Wettbewerb, DG Umwelt etc. Die anderen Generaldirektionen wie zum Beispiel DG Übersetzung, J uristischer Dienst, das Generalsekretariat, DG Budget, Eurostat etc. haben keine direkten Politikentwicklungsaufgaben und sind daher für die Forschungsfrage irrel evant. Durch eine genaue Durchsicht der Organigramme konnten alle $A L$ in den General direktionen mit Politikentwicklungsaufgaben, die sel bst nachweislich Politikgestaltungsaufgaben ausüben, identifiziert werden. Aus dieser Grundmenge wurden 200 Individuen zufällig ausgewählt. 116 Telefoninterviews kamen zustande - 58 \% Rücklaufquote. Die Interviews wurden auf Englisch und Französisch in zwei Wellen - März und Oktober 2006 - geführt. Vgl. die Ergebnisse und Details zur Durchführung Bauer (FN 22).

36 Auf drei gestellte Fragen ist kurz näher einzugehen. Eine zentrale Frage lautete, welche „Position“ am meisten durch die Reform verändert wurde. Zweidrittel der AL
} 
Man kann aus dieser Perspektive die Kinnock Reform fol gendermaßen bewerten: Wahrscheinlich erfüllt die Reform ihre primären Ziele. Die eingeführten Elemente des New Public Management schaffen mehr Transparenz an der politischen und administrativen Spitze der Organisation. Möglich werden diese Veränderungen allerdings durch eine umfassende Redefinition der Rolle der Abteilungsleiter. Sie müssen die Neuerungen anwenden und in einem komplexen Verwaltungsprozess umsetzen. Die Abteilungsleiter selbst bevorzugen mehrheitlich jedoch ihre „alte“ Rolle als politische Unternehmer und Policy-Innovatoren und sehen diese ihre „alte“ Rolle durch die jüngsten Veränderungen, die die Kinnock Reform bringt, als nicht mehr optimal ausfüllbar $a^{37}$. Kurzum, die Verwandlung der Rolle des Abteilungsleiters von einem pro-aktiven Politikexperten in einen reaktiven Prozessmanager schränkt deren individuelles Potenzial als politische Unternehmer zu agieren, nachhaltig ein. Die Kommission als Organisation dürfte also durch die jüngste Reform an konkretem Politikgestaltungspotenzial verlieren. Der Policy-Output der Kommission wird also durch die KinnockReformen offensichtlich höchst negativ beeinflusst.

\section{Fazit}

Das zentrale Argument dieses Aufsatzes ist, dass es möglich und sinnvoll ist, über die Konzentration auf den Nexus zwischen Throughput-Änderungen und Output-Resultaten den Theoriegehalt von Verwaltungsreformanalysen zu erhöhen. Die Frage am Beginn einer Verwaltungsreformanalyse sollte lauten, was ist aus der Perspektive des individuellen Forschungsinteresses die zentrale Funktion einer bestimmten Verwaltung und wie verändern die interessierenden Reformen des Apparats die organisatorische Wahrnehmung dieser Funktion? Was also sind die beobachtbaren Implika-

sehen ihre eigene Rolleals die Position an, die am meisten verändert wurde. Diezweite zentrale Frage zielte darauf, die Affinität der AL zu ihrer potenziellen Rolle als Politikunternehmer zu messen. Die Frage stammt von L. Hooghe (FN 22) und lautet, "Should the brightest policy innovators get better career prospects?" Zweidrittel der $\mathrm{AL}$ stimmen dieser Frage zu; sie sehen sich am liebsten also nach wie vor als Politikentwicklungsexperten und nicht als Manager. Ferner wurde nach den konkreten Auswirkungen der Reform im individuellen Arbeitsbereich gefragt. Dabei sagen mehr als zweidrittel der AL, dass im Vergleich mit der Zeit vor der Reform die Effizienz der Abteilung gesunken sei, sich das Personalmanagement verschlechtert habe, das es keine klaren Vorgaben von den Vorgesetzten gebe, keine größere Selbständigkeit mehr möglich sei und insgesamt ein größerer Bürokratieaufwand betrieben werden müsse. Das sind nicht irgendwelche Fragen, sondern diese Fragen entstammen den Reformweisbüchern und waren explizite Verbesserungsziele. Auf dieser generellen Ebene demonstriert der Survey also eine sehr große Ablehnung bzw. Skepsis gegenüber der Kinnock Reform unter den $\mathrm{AL}$.

37 Als "gute Beamte" sehen die AL aber, dass - aus der Perspektive ihrer neuen Managementaufgaben! - verschiedene Elemente durchaus Sinn machen und unterstützen diese auch mit relativer oder sogar absoluter Mehrheit. 
tionen von Organisationswandel und wie wirken sich diese Veränderungen nach dem disziplinären Wissensstand auf den Throughput-Output-Nexus aus?

Das Beispiel der Analyse der Verwaltungsreform der Europäischen Kommission verdeutlicht, wie über eine derartige ex-ante Theoretisierung und strikte Konzentration auf den Throughput-Output-Nexus, Aussagen über Organisationsreform und Reformresultat möglich werden. Mit dem Blick auf die Bewertung der Kommissionsreform mag das Ergebnis nur einen bescheidenen Fortschritt darstellen. Auf der Grundlage dieser Analyse kann aber nun die Hypothese formuliert werden, dass Organisationsveränderungen im Sinne von New Public Management in Verwaltungseinheiten, die sich über fachspezifische Expertise und dezentrale Politikgenerierung definieren, eine Qualitätsminderung im Hinblick auf den erzielbaren PolicyOutput mit sich bringen. Eine Interpretation könnte nun sein, dass New Public Managementreformen eher in Kontexten eingesetzt werden sollten, die eine relativ niedrige Kreativität der Mitarbeiter erfordern und eher Routineverwaltungshandeln betreffen. New Public Managementreformen haben also für „höhere“ Verwaltungseinheiten, die komplexe Prozesse der inter-organisationalen Politikinnovation und Systementwicklung steuern oder moderieren, äußerst negative Folgen.

Für die Argumentation über die Bedeutung des Reform-Ergebnis-Zusammenhangs ist dieses konkrete Resultat allerdings zweitrangig. Wichtiger ist, dass es sich hierbei um eine Hypothese handelt, die offen für eine empirische Widerlegung ist. Künftige Analyse zu Verwal tungsreformen im Kontext des Mechanismus „politisches Unternehmertum“ - beispiel sweise Unterorganisationen der Vereinten $\mathrm{N}$ ationen, der Weltbank oder des Nordischen Rates ${ }^{38}$ könnten die unterstellten Zusammenhänge weiter konkretisieren, abändern oder ganz verwerfen. In dieser mögl ichen Falsifizierbarkeit besteht aber gerade das Potenzial der fallübergreifenden Wissensakkumulation und der Steigerung der Theoriefähigkeit verwaltungswissenschaftlicher ReformanaIysen $^{39}$. Durch die vorgeschlagene Untersuchungsstrategie scheint al so eine bescheidene ex-ante Theoretisierung der verwaltungswissenschaftlichen Forschungsfrage mithilfe policyanalytischer Konzeptionalisierungen möglich, die den Theoriegehalt und die theoretische Anschlussfähigkeit von Reformanalysen in der Teildisziplin Verwaltungswissenschaft durchaus zu erhöhen vermag.

38 Siehe Bauer / Knill (FN 29).

39 J edenfalls gilt dies sofern man einem kritisch-rationalistischen Wissenschaftsverständnis zuneigt. K. Popper, Logik der Forschung, in H. Keuth (Hrsg.), 2004; I. Lakatos, Falsifikation und die Methodologie wissenschaftlicher Forschungsprogramme, in: ders. / A. Musgrave (H rsg.), K ritik und Erkenntnisfortschritt, 1974, S. 89. 


\begin{abstract}
Clarifying the relationship between organisational reform and reform outcome is a central objective of students of public administration or administrative sciences. The last decades have witnessed intensive research efforts in this area. However, the added value achieved in theory development remains meagre, at best. This article argues that by systematically incorporating policy analysis conceptualisations, the administrative sciences can reduce this deficit and generate more advanced theoretical knowledge. By referring to the recent reforms of the E uropean Commission, this article illustrates the potential of an approach that systematically combines policy analysis insights and public administration research interests. Public admi nistration reform scholars are advised to concentrate their analysis on the nexus between organisational reform and reform output if their aim is to steadily improve the capacity for theory development within this sub-discipline of public administration.
\end{abstract}

